Indexed by

\title{
Scopus
}

\section{BASIC MATHEMATICAL FORM OF MICHELL STRUCTURE}

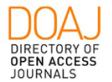

Crossref

\section{Sanaullah Khushak}

Harbin Engineering University, College of Mechanical and Electrical Engineering,

Harbin, China

\section{Asif Raza}

Harbin Engineering University,

College of Mechanical and

Electrical Engineering,

Harbin, China

\section{Ani Luo}

Harbin Engineering University, College of Mechanical and Electrical Engineering,

Harbin, China

\author{
Muhammad Basit Chandio \\ Harbin Engineering University, \\ College of Mechanical and \\ Electrical Engineering, \\ Harbin, China
}

KOBSON

Key words: force density, mathematical form, Michell structure, minimal mass, tensegrity structure

\section{Cite article:}

Sanaullah, K., Ani, L., Muhammad, B. C., \& Asif, R.[2021]. Basic mathematical form of michell structure. Journal of Applied Engineering Science, 19(2), 547 - 550. DOI:10.5937/jaes0-27675

Online aceess of full paper is available at: www.engineeringscience.rs/browse-issues 


\title{
BASIC MATHEMATICAL FORM OF MICHELL STRUCTURE
}

\author{
Sanaullah Khushak*, Ani Luo, Muhammad Basit Chandio, Asif Raza \\ Harbin Engineering University, College of Mechanical and Electrical Engineering, Harbin, China
}

Michell structure is well known among tensegrity structures due to its optimization form and minimum mass of the structure. Michell had adopted this idea from the results of James C Maxwell's research on truss design. This paper presents the basic mathematical model of Michell structure based on complexity order $q=2$ in the two-dimensional coordinate system. This mathematical model imparts the analysis of all nodes and all members of Michell structure and investigates their position to construct a stable Michell structure. This basic mathematical model of Michell structure of complexity order $q=2$ can be used as a foundation to develop the Michell structure of high complexity orders. Furthermore, the force density in each member of the structure has been studied. An expression to calculate the minimum mass of structure has been defined at the end of this paper, which is the most important factor to construct any kind of tensegrity structure.

Key words: force density, mathematical form, Michell structure, minimal mass, tensegrity structure

\section{INTRODUCTION}

In 1962, Fuller coined the word tensegrity by the consolidation of two words 'tension' and 'integrity' [1]. Tensegrity structures are well known due to their stability, self-equilibrium, and form finding analysis [2]. These structures are composed of compressive parts; known as bars, and the tensile parts; known as strings. The bar elements are subjected to compressive force and string elements are subjected to tensile force[3].

Numerous methods have been introduced to build simple units in order to achieve complex structures. The applications of tensegrity structure are found in various fields as in robotics, civil engineering, medical and aerospace due to its properties of light weight and self-equilibrium capability [4]. The most fundamental requirement to design any structure is structural optimization approach. The basic parameters for structural design of any structure are its shape and size [5]; these parameters determine the minimum weight of structure by considering its self-stable equilibrium conditions under external force.

Michell presented the theory for such structure in 1904 and are known as Michell structure. He studied the cantilever truss of optimal design to transmit the applied load to the given fixed point of support[6]. Although Michell structure is composed of bars and strings but it is quite different from other tensegrity structures. Michell structure contains equal number of bars and strings. The number of elements in this structure depends upon the order of complexity. Many researchers have presented the layout of least weight theory and some shortcomings of Michell structure theory [7]. In this article, we have developed the basic layout of the Michell structure based on complexity order $q=2$. This method will be supportive for new learners to develop Michell structure of any higher complexity order.

\section{DESIGN OF STRUCTURE AND PARAMETERS}

Design of any tensegrity structure depends on nodes, bars, strings and various angles between members but design of Michell structure needs complexity order $q$ as an additional parameter. This section of paper describes these parameters.

Michell structure has fixed outermost radius $r_{0}$ and all other radii are dependent on this fixed radius. Two angles $\varnothing$ and $\beta$ are constant and the length $I_{0}$ and $I_{1}$ between their respective nodes can be changed by changing the radius of structure as shown in fig.1.

Complexity order $q$ and two angles $\varnothing \& \beta$ are required to develop Michell Structure; where $\varnothing$ is the angle between the two adjacent radii and beta $\beta$ is an angle between the compressive and tensile members in the finite complexity. Value of $2 \beta$ must be always less than 90 degree, if it approaches to 90 degree then complexity will reach to infinity[3]. The sin functions of angles $\varnothing \& \beta$ are used to find the two constants $\alpha$ and $c$ by using Eq. (1). These constants $\alpha$ and $c$ can be used to calculate the inner radii

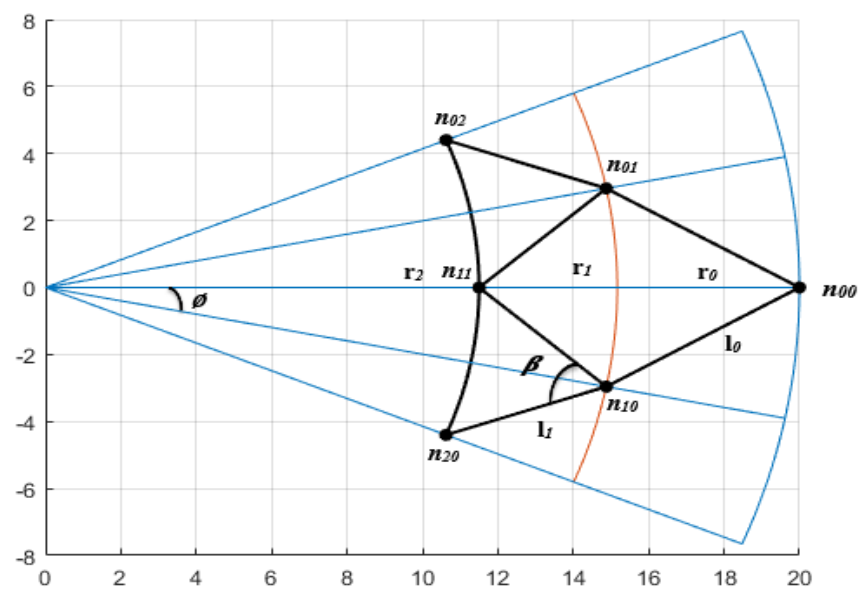

Figure 1: Michell structure of complexity order 2 
and length of the element between two nodes by using Eq. (2) and Eq. (3) respectively.

$\alpha=\frac{\sin \beta}{\sin (\beta+j)} \quad c=\frac{\sin j}{\sin (j+\beta)}$

$r_{j+1}=\alpha r_{j} j=0,1 \ldots q$

$p_{j}=c r_{j} \quad j=0,1 \ldots q$

Total number of nodes $t_{n}$ and total number of members $t_{m}$ in Michell structure can be determined by Eq. (4) and Eq. (5) respectively.

$t_{n}=\frac{(q+1)(q+2)}{2}$

$t_{m}=[q(q+1)]$

Where q denotes the complexity order of Michell structure.

The structure of complexity order $q=2$ has 6 nodes and 6 members. It is hard to recognize the bar members and string members in Michell structure because this structure of any complexity order has equal number of bars and strings as shown in fig 1 . The answer of this question depends upon the direction of an applied external force [2]. If the external force is being applied in downward direction at 90 degree then red lines will represent the strings and blue lines will indicate the bars as shown in fig. 2 (a), and if external force is applied in upward direction then blue lines will represent the bars and red lines will indicate the strings as demonstrated in fig.2 (b)

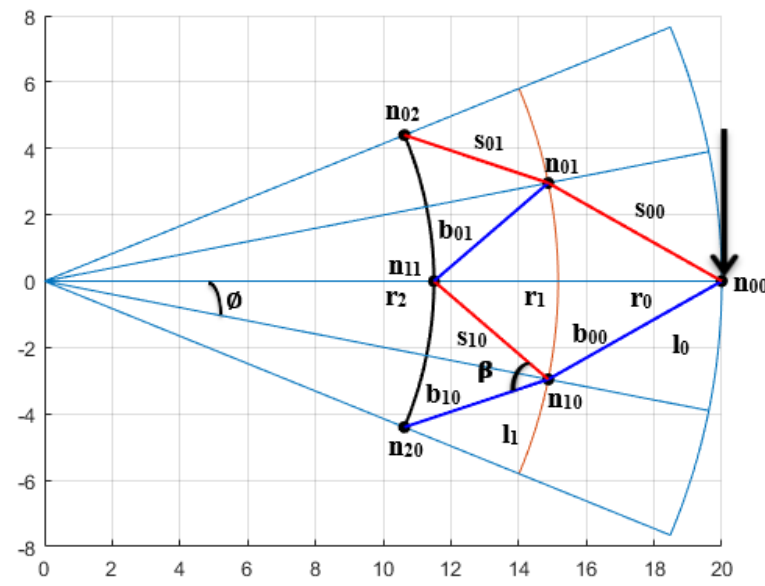

(a)

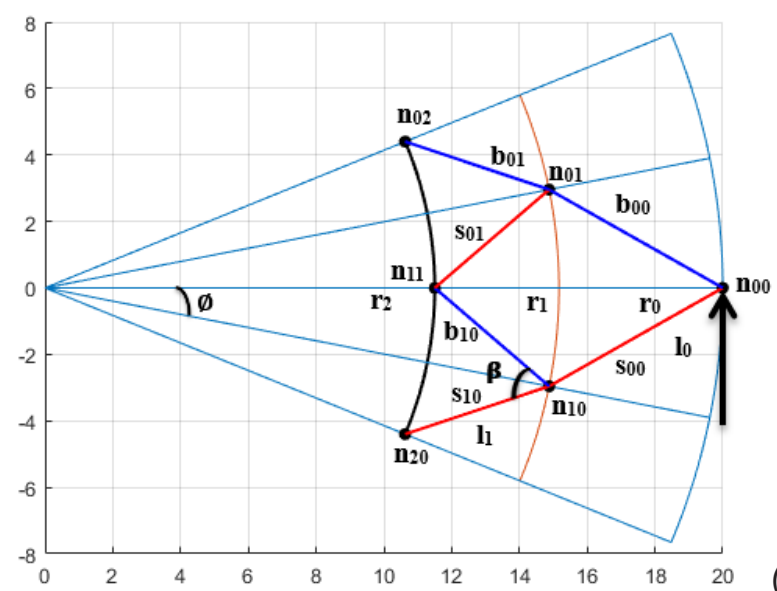

Figure 2: External force acting in downward direction (a) External force acting in upward direction (b)
We consider fig.2 (a) to analyze the string matrix, bar matrix, connectivity matrices and force density of structure.

\section{Nodes and node matrix}

The Michell structure of complexity order $q$ will have $q+1$ spiral and each spiral has $q-i+1$ node. Node matrix $N$ is given in Eq. (6).

$$
\begin{aligned}
& N=\left[\begin{array}{lllll}
N_{0} & N_{1} & N_{i} & \cdots & N_{q}
\end{array}\right], i=0,1, \ldots q \\
& N_{i}=\left[\begin{array}{llll}
n_{i 0} & n_{i 1} & \cdots & n_{i(q-i)}
\end{array}\right]
\end{aligned}
$$

Where $N_{i}$ denotes the spiral.

Position of each node can be determined by using radius $r$ and trigonometric functions of $\varnothing$ as mentioned in Eq. (8)

$n_{i j}=\left[r_{j} \cos (j-i) j \quad r_{j} \sin (j-i) j\right], j=0,1, \ldots q-1$

Where $i$ denotes the spiral and $j$ denote the radius where node lies; and both subscripts together denote the node number. The nodes of the Michell structure of complexity order 2 can be written in matrix form as

$N=\left[\begin{array}{lll}N_{0} & N_{1} & N_{2}\end{array}\right]$

$N=\left[\begin{array}{llllll}n_{00} & n_{01} & n_{02} & n_{10} & n_{11} & n_{20}\end{array}\right]$

The mirror image of all lines from $x$-axis will create the reflection of all nodes and lines; these reflected nodes have same position and magnitude and lie on their same respective radii as shown in fig. 1

$\left\|n_{i(j+1)}\right\|=\left\|n_{(i+1) j}\right\|$

\section{String matrix and string connectivity matrix}

Michell structure of complexity order 2 has three strings and three bars. Each member is connected by two nodes[8]. Eq. (11) shows the string matrix, Eq. (12) shows the string element on the each spiral and Eq. (13) show the string vector, whereas Eq. (14) expresses the connectivity matrix of string $C_{S}$.

$S=\left[\begin{array}{llllll}S_{0} & S_{1} & \cdots & S_{i} & \cdots & S_{q-1}\end{array}\right]$

$S_{i}=\left[\begin{array}{llllll}s_{i 0} & s_{i 1} & \cdots & s_{i j} & \cdots & s_{i(q-i-1)}\end{array}\right]$

$S_{i j}=\left[n_{i(j+1)}-n_{i j}\right]$

Where $N_{i(j+1)}$ shows the starting point and $n_{i j}$ denote the ending point string

$c_{s}=\left[\begin{array}{cccccc}-1 & 1 & 0 & 0 & 0 & 0 \\ 0 & -1 & 1 & 0 & 0 & 0 \\ 0 & 0 & 0 & -1 & 1 & 0\end{array}\right]$

The node of starting point and the node of end point of each member are denoted 1 and -1 respectively; and all the other nodes that do not touch the member are denoted by zero. The sum of each row must be zero. Both connectivity matrices of bars and strings of Michell structure 
of complexity order 2 will be order of $3 \times 6$. The number of rows shows the total number of bars and number of columns shows the total number of nodes. The relationship with string matrix and node matrix can be defined as:

$S=N C_{S}^{T}$

$C_{T}^{S}$ denotes the transpose of the connectivity matrix of strings.

\section{Bar matrix and bar connectivity matrix}

The number of bars in a Michell structure is equal to the number of strings. Eq. (16), (17), (18) and (19) represent the bar matrix, bar elements on each spiral, bar vector and bar connectivity matrix respectively,

$B=\left[\begin{array}{llllll}B_{0} & B_{1} & \cdots & B_{i} & \cdots & B_{q-1}\end{array}\right]$

$B_{i}=\left[\begin{array}{llllll}b_{i 0} & b_{i 1} & \cdots & b_{i j} & \cdots & b_{i(q-i-1)}\end{array}\right]$

$b_{i j}=\left[n_{(i+1) j}-n_{i j}\right]$

Where $n_{(i+1) j}$ shows the starting point and $n_{i j}$ denote the ending point bar.

$C_{B}=\left[\begin{array}{cccccc}-1 & 0 & 0 & 1 & 0 & 0 \\ 0 & 0 & 0 & -1 & 0 & 1 \\ 0 & -1 & 0 & 0 & 1 & 0\end{array}\right]$

The relationship between bar matrix and node matrix can be found by following Eq.

$B=N C_{B}^{T}$

Where $C_{B}{ }^{\top}$ is the transpose of the connectivity matrix of bars.

Connectivity matrix $\mathrm{C}$ of whole structure as mentioned in

Eq. (21) can be obtained by combining the Eq. (14) \& (19),

$$
C=\left[\begin{array}{cccccc}
-1 & 1 & 0 & 0 & 0 & 0 \\
0 & -1 & 1 & 0 & 0 & 0 \\
0 & -1 & 0 & 0 & 1 & 0 \\
-1 & 0 & 0 & 1 & 0 & 0 \\
0 & 0 & 0 & -1 & 0 & 1 \\
0 & 0 & 0 & -1 & 1 & 0
\end{array}\right]
$$

\section{FORCE DENSITY ANALYSIS}

An important factor related to tensegrity structures is force density[9]. Force density is "force per unit length of member" and can be formulated as Eq. (22).

$\lambda_{i, j}=\gamma_{i, j}=\frac{f_{i, j}}{I_{i, j}}$

$\lambda_{i . j}=$ force density of bar

$\gamma_{i . j}=$ force density of string

$f_{i . j}=$ internal force from i (starting node) to $\mathrm{j}$ (end node)

$I_{i . j}=$ length from $i$ (starting node) to $j$ (end node)

The general form of force density Equation in two-dimensional system can be written as:

$\sum_{r=N} \frac{x_{i}-x_{j}}{l_{i, j}} f_{i j}=\sum_{r=N}\left(x_{i}-x_{j}\right) \lambda_{i, j}=\sum_{r=N}\left(x_{i}-x_{j}\right) y_{i, j}$
$\sum_{r=N} \frac{y_{i}-y_{j}}{l_{i, j}} f_{i, j=} \sum_{r=N}\left(y_{i}-y_{j}\right) \lambda_{i, j}=\sum_{r=N}\left(y_{i}-y_{j}\right) v_{i, j}$

Negative sign (-) is used for the elements in compression and positive sign (+) is used for elements in tension for force density analysis.

We take node $n_{00}$ to analyze force density on this node. One end of string $s_{00}$ and bar $b_{00}$ is connected with a common node $n_{00}$ and other end of both members is connected with nodes $n_{01} \& n_{10}$ respectively. The nodes $n_{01}$ \& $n_{10}$ lie on the same radius, so length of string $s_{00}$ and bar $b_{00}$ is same as demonstrated in Fig 2 (a). Relationship between force density of string $s_{00}$ and bar $b_{00}$ on node $n_{00}$ along $x$-axis can be calculated by using the Eq. (23) as follows

$\sum f_{x}=0$

$\sum f_{01.00} \frac{\left(n_{01 x}-n_{00 x}\right)}{l_{01.00}}+f_{10.00} \frac{\left(n_{10 . x}-n_{00 x}\right)}{l_{10.00}}=0$

$\lambda_{01.00}=\gamma_{01.00}=\frac{f_{01.00}}{l_{01.00}}$ or $\quad \lambda_{10.00}=\gamma_{10.00}=\frac{f_{10.00}}{l_{10.00}}$

$\gamma_{01.00}\left(n_{01 x}-n_{00 x}\right)+\lambda_{10.00}\left(n_{10 x}-n_{00 x}\right)=0$

$\gamma_{10.00}=-\frac{\lambda_{10.00}\left(n_{10 x}-n_{00 x}\right)}{\left(n_{01 x}-n_{00 x}\right)}$

Similarly, relationship between force density of string $s_{00}$ and bar $b_{00}$ on node $n_{00}$ along y-axis can be calculated by Eq. (23.1) as follows

$\sum f_{\mathrm{y}}=0$

$\sum f_{01.00} \frac{\left(n_{01 y}-n_{00 y}\right)}{l_{01.00}}+f_{10.00} \frac{\left(n_{10 y}-n_{00 y}\right)}{l_{10.00}}=0$

$V_{01.00}\left(n_{01 y}-n_{00 y}\right)+\lambda_{10.00}\left(n_{10 y}-n_{00 y}\right)=0$

$\lambda_{10.00}=-\frac{V_{01.00}\left(n_{01 y}-n_{00 y}\right)}{\left(n_{10 y}-n_{00 y}\right)}$

Force density of string $s_{00}$ and bar $b_{00}$ on node $n_{00}$ has been analyzed. Same method can be used to analyze the force density of remaining members on other remaining nodes.

\section{EQUILIBRIUM EQUATION}

As we know that $\gamma$ denotes force density of strings and strings carry tensional force. $\lambda$ denotes the force density of bars and bars carry compressive force. The equilibrium equation of internal forces of structure can be written as

$S y C_{S}-B \lambda C_{B}=0$

If an external force $\mathrm{W}$ is applied on the structure then Eq. (28) becomes

$W=S \gamma C_{S}-B \lambda C_{B}$

Eq. (29) is general form of system. S and B denote the string vector and bar vector respectively and their values 
can be taken from Eq. (15) \& (20).

As we have already mentioned that Michell structure of complexity order 2 has three strings and three bars, so values of their internal forces in diagonal matrices is

$$
\gamma=\left[\begin{array}{ccc}
\gamma_{00} & 0 & 0 \\
0 & \gamma_{01} & 0 \\
0 & 0 & \gamma_{10}
\end{array}\right] \quad \lambda=\left[\begin{array}{ccc}
\lambda_{00} & 0 & 0 \\
0 & \lambda_{01} & 0 \\
0 & 0 & \lambda_{10}
\end{array}\right]
$$

By inserting the values of $C_{S} \& C_{B}$ in Eq. (29), we get external force Equation as:

$$
\begin{gathered}
W=\left[\begin{array}{lll}
s_{00} & s_{01} & s_{10}
\end{array}\right]\left[\begin{array}{ccc}
V_{00} & 0 & 0 \\
0 & V_{01} & 0 \\
0 & 0 & \gamma_{10}
\end{array}\right]\left[\begin{array}{cccccc}
1 & -1 & 0 & 0 & 0 & 0 \\
0 & 1 & -1 & 0 & 0 & 0 \\
0 & 0 & 0 & 1 & -1 & 0
\end{array}\right] \\
-\left[\begin{array}{lll}
b_{00} & b_{01} & b_{10}
\end{array}\right]\left[\begin{array}{ccc}
\lambda_{00} & 0 & 0 \\
0 & \lambda_{01} & 0 \\
0 & 0 & \lambda_{10}
\end{array}\right]\left[\begin{array}{cccccc}
1 & 0 & 0 & -1 & 0 & 0 \\
0 & 0 & 0 & 1 & 0 & -1 \\
0 & 1 & 0 & 0 & -1 & 0
\end{array}\right]
\end{gathered}
$$

By multiplying the vector-matrix with corresponding force density matrix and connectivity matrix we will get external force on each node of structure [10]. The external force in vector form can be written as:

$W=\left[\begin{array}{lll}w_{1} & \cdots & w_{n}\end{array}\right]$

\section{MINIMUM MASS}

Michell structure is subjected to pure bending only rather than yielding or buckling failure. Yielding and buckling failure can occur in other types of tensegrity structure. Therefore, mass of these type of tensegrity structures (except Michell structure) can be calculated on the bases of their yielding and buckling constraints, while Michell structure has a pure bending form of failure (if occurs) only; therefore, the minimum mass of this structure can be calculated by taking the "sum of the square of all bars and strings multiplied by the corresponding force density unit."

$$
M=\sum_{N=q}\left(\gamma_{i j}\left\|S_{i j}\right\|^{2}+\lambda_{i j}\left\|b_{i j}\right\|^{2}\right)
$$

Where $\left\|s_{i j}\right\| \&\left\|b_{i j}\right\|$ denotes the string and bar members[11] respectively, $N$ denote the node matrix and $q$ denote the complexity order.

\section{CONCLUSION}

Michell structure of complexity order $q=2$ has been analyzed. Node matrix, bar matrix, string matrix and their connectivity matrices were developed. Force density in elements has been discussed. Equilibrium Equation of structure has been derived. Minimal mass of Michell structure of complexity order 2 has been found. Michell structure of any higher complexity order can be easily developed by using this methodology of complexity order 2 .

\section{ACKNOWLEDGMENT}

This work was supported by the National Natural Science Foundation of China (Grant Nos. 51605111, 51675114 and 51875111).

\section{REFERENCES}

1. Rene, M. (2003). Tensegrity Structural Systems for the Future. pp.7-16.

2. Jing, Y, Z., Makoto, O. (2015). Tensegrity Structures, Form, Stability, and Symmetry. Springer, vol 6, pp 1-11. Doi. 10.1007/978-4-431-54813-3

3. Robert, E, S., Mauricio, C, d, O. (2009). Tensegrity Systems. Springer, New York. pp 1-43, 129-154. Doi. 10.1007/978-0-387-74242-7

4. Heping, L., Jingyao, Z., Makoto, O. (2018) .3bar tensegrity units with non-equilateral triangle on an end plane. vol. 92, pp. 124-130. https://doi. org/10.1016/j.mechrescom.

5. Dhanjoo, G, N., Meyer M, R. (1968). Development of Michell Minimum Weight Structures.

6. Prager, W. (1977). Optimal Layout of Cantilever Trusses. Journal of Optimization Theory and Applications, vol. 23, no. 1.

7. Rozvany, G, I, N. (1996). Some shortcomings in Michell's truss theory. Springer, vol. 12, p. 6,

8. Muhammad, B, C., Ani, L., Yaohui, L., Sanaullah, K., Asif, R. (2019). The Dynamic similarity of Six Bar Ball Tensegrity Structure in Compression and Expansion Processes. Journal of Harbin Institute of Technology. (New Series). Doi: 10.11916/j. issn.1005-9113.2019032.

9. Guest, S, D., Koohestani, k. (2013). A new approach to the analytical and numerical form-finding of tensegrity structures. International Journal of Solids and Structures. Vol. 50, issue 19. doi.10.1016/ijsolstr.2013.05.014.

10. Ani, L., Robort, E, S., Heping, L., Rongqiang, L., Hongwei, G., Longkun, W. (2014). Structure of the ball tensegrity robot. IEEE.

11. Nagase, k. Robert, E, S. (2014). Minimal Mass Design of Tensegrity Structures, Journal of the International Association for Shell and Spatial Structures. vol.55, no. 1 . 